Christophe Lefebvre Aurélie Tasiemski Michel Salzet

\section{ADRESSE}

C. Lefebvre, A. Tasiemski, M. Salzet : Laboratoire d'endocrinologie des annélides, UPRESA Cnrs 8017, Bâtiment SN3, Université des sciences et technologies de Lille, 59655 Villeneuve-d'Ascq Cedex, France.

$\mathrm{m} / \mathrm{s} n^{\circ} 2$, vol. 16 , février 2000

\title{
Peptides opioïdes, substances opiacées et réponse immunitaire
}

Chez les invertébrés comme chez les vertébrés, les peptides opioïdes et les substances opiacées interviennent soit dans la mise en route, soit dans l'inhibition des réponses immunitaires innée et spécifique. Ces analogies constatées entre invertébrés et vertébrés indiquent l'apparition d'une stratégie immunitaire efficace et perfectionnée très tôt au cours de l'évolution. Les fonctions de régulation impliquant les peptides opioïdes et les substances opiacées représentent donc un patrimoine vieux de $\mathbf{6 0 0}$ millions d'années particulièrement performant puisqu'il est conservé jusqu'à l'homme. Toutefois, la complexité des systèmes nerveux et immunitaire chez les mammifères rend l'étude de ces mécanismes fondamentaux particulièrement difficile. Une approche alternative consiste à développer des modèles d'étude invertébrés plus simples, afin de mieux comprendre les processus mis en jeu lors de la réponse innée, dont les signaux sont à l'origine de la réponse adaptative chez les vertébrés.

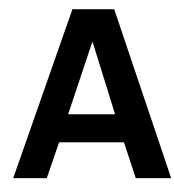

$\mathrm{u}$ XIX $^{\mathrm{e}}$ siècle, toutes les fonctions et manifestations physiologiques, parmi lesquelles les réactions immunitaires, étaient considérées comme étant sous le contrôle du cerveau, organe central assurant l'homéostasie de l'organisme face aux maladies. Par la suite, l'immunologie a connu un essor considérable avec les découvertes d'Élie Metchnikoff sur la phagocytose et celles de Louis Pasteur sur la vaccination. Dès lors, chercheurs et médecins ont mieux perçu l'importance du système immunitaire dans la réponse de l'organisme face aux agressions étrangères. Il a cependant fallu attendre la seconde moitié de notre siècle pour que la question des interconnexions possibles entre systèmes nerveux et immunitaire soit remise au goût du jour.

$\mathrm{Au}$ cours de ces quinze dernières années, plusieurs travaux ont montré que des modifications de l'état mental (stress, hypnose) influencent la réponse immunitaire en modulant la résistance de l'organisme aux maladies (figure 1). Réciproquement, le système immunitaire est susceptible d'activer des voies périphériques (nerf vague) et centrales afin de produire, par exemple, l'état fiévreux qui accompagne une infection. De nombreux travaux ont confirmé l'exis- 
tence d'échanges d'informations bidirectionnels entre les systèmes neuroendocrinien et immunitaire via des messagers intercellulaires [1]. Ces relations font intervenir différentes voies nerveuses, l'axe hypothalamohypophyso-surrénalien et la voie sanguine. A l'intérieur de ces voies, différentes molécules peuvent être impliquées parmi lesquelles des peptides (CRH: corticolibérine, ACTH: hormone adrénocorticotrope), des monoamines (noradrénaline, adrénaline et dopamine), des glucocorticoïdes, des cytokines (interleukines-1 et -6 ; tumor necrosis factor $\alpha$ ), des radicaux libres médiateurs comme le monoxyde d'azote (NO) [2], des pep-

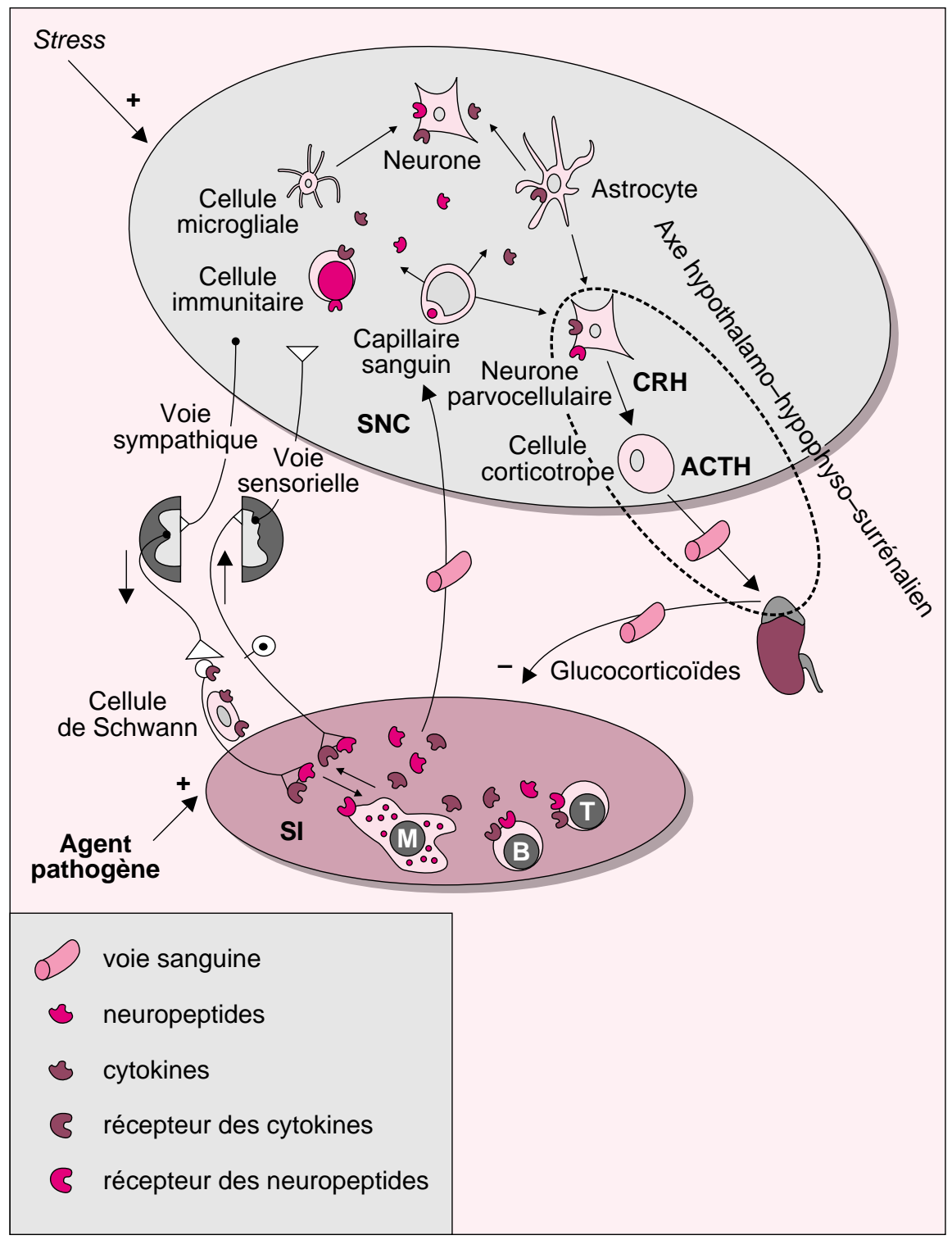

Figure 1. Interactions entre systèmes nerveux, endocrinien et immunitaire chez les mammifères [1]. Dans le cadre de ces échanges d'informations, le système nerveux peut exercer un contrôle sur le système immunitaire via entre autres l'axe hypothalamo-hypophyso-surrénalien. À l'inverse, la réponse immune peut alerter le cerveau afin de déclencher certains processus comme la fièvre. Ces interactions impliquent certains neuropeptides et cytokines. Il est intéressant de noter que quelques membres de ces deux familles de molécules ont été retrouvés à la fois dans le système nerveux et dans le système immunitaire, ce qui renforce l'idée que neuropeptides et

tides opioïdes et des substances opiacées (morphine, anandamide). Ces deux dernières familles de molécules, longtemps étudiées pour leur rôle analgésique, jouent également un rôle dans la modulation de la réponse immune.

L'ensemble de ces résultats a conduit à l'émergence d'un nouveau domaine de recherche à la confluence de plusieurs disciplines telles que l'immunologie, la neurobiologie, l'endocrinologie et même la psychiatrie. Ce domaine constitue désormais la neuro-immunologie, ou psychoneuro-immunologie lorsque le sujet d'étude est l'homme. Cependant, l'analyse détaillée de ces voies de communication est rendue particulièrement difficile par la complexité des systèmes nerveux et immunitaire des vertébrés supérieurs. Une approche alternative consiste à utiliser un modèle d'étude plus simple possédant néanmoins les mécanismes fondamentaux présents chez les mammifères. Les nombreux travaux réalisés chez Drosophila melanogaster, Caenorhabditis elegans, Aplysia californica ou Hirudo medicinalis ont démontré l'intérêt des modèles invertébrés dans des domaines aussi variés que ceux du développement, de l'immunité, de l'apoptose, du vieillissement ou de la neurobiologie. Dans cet article, nous aborderons la comparaison des mécanismes moléculaires mis en jeu chez les vertébrés et les invertébrés au cours des interactions entre le système immunitaire, les peptides opioïdes et les substances opiacées.

\section{Implication des peptides opioïdes dans la réponse immunitaire}

Ces molécules, présentes chez les vertébrés, sont issues de trois précurseurs: la pro-opiomélanocortine (POMC), la pro-enképhaline et la pro-dynorphine, toutes trois localisées dans le cerveau [3]. La majorité des études concernant les peptides opioïdes chez les vertébrés ont porté sur leur localisation neuroanatomique, leur caractérisation biochimique et moléculaire et leur biosynthèse. A l'inverse, les données concernant ces précurseurs et leur métabolisme au niveau du système immunitaire sont à la fois récentes et 
fragmentaires (figure 2). Ainsi, l'existence d'ARN messager codant pour la POMC a pu être démontrée au niveau des macrophages pulmonaires et de la pulpe rouge de la rate [5]. La pro-enképhaline est quant à elle retrouvée dans les lymphocytes $\mathrm{T}$ et les monocytes [6].

$\mathrm{Au}$ niveau du système nerveux, ces précurseurs subissent une maturation sous l'effet de pro-hormones convertases (PC) spécifiques [7]. Certaines de ces enzymes telles que la PC3 sont co-localisées avec la proenképhaline dans les macrophages humains [8], ce qui suggère l'existence de mécanismes de maturation semblables dans le système immunitaire et le système nerveux central. Ces travaux tendent à montrer que les précurseurs sont présents dans les cellules immunitaires et que celles-ci sont capables de produire les principes actifs. La présence et l'action des peptides opioïdes au niveau du système immunitaire peuvent donc avoir deux origines, l'une dans les cellules immunitaires (modulation autocrine et paracrine) et l'autre dans le système nerveux (modulation endocrine). Les différents exemples présentés dans cet article ne nous permettent pas de trancher entre ces deux hypothèses. En revanche, il est indéniable que les peptides opioïdes modulent la réponse immunitaire.

\section{La majeure partie des données} et la littérature porte sur les dérivés de la POMC et de la pro-enképhaline

En général, on associe les dérivés de la POMC [9] avec un rôle inhibiteur de la réponse inflammatoire et ceux de la pro-enképhaline [10] avec un rôle activateur. Cependant, si les données récentes obtenues pour la proenképhaline confirment cette activité, il n'en est pas de même pour la POMC. La POMC contient entre autres deux molécules capables de moduler le système immunitaire. Or, si certains travaux font état de l'action immunosuppressive de l'hormone mélanostimulante $(\alpha-\mathrm{MSH}, \alpha$ melanocyte stimulating hormone) sur les monocytes [11], la $\beta$-endorphine possède un effet opposé. Elle est libérée par de nombreuses cellules immunitaires telles que les lymphocytes et les macrophages lors d'une agression bactérienne, et son action rejoint celle de la méthionine-enképhaline (Met-Enk), issue de la maturation de la pro-enképhaline [3]. En effet, chez la souris, l'injection de Met-Enk entraîne une augmentation de la prolifération des lymphocytes $\mathrm{B}$ et $\mathrm{T}$ [12]. Chez l'homme, l'injection de leucine-enképhaline (Leu-Enk), un peptide opioïde également issu de la maturation de la pro-enképhaline

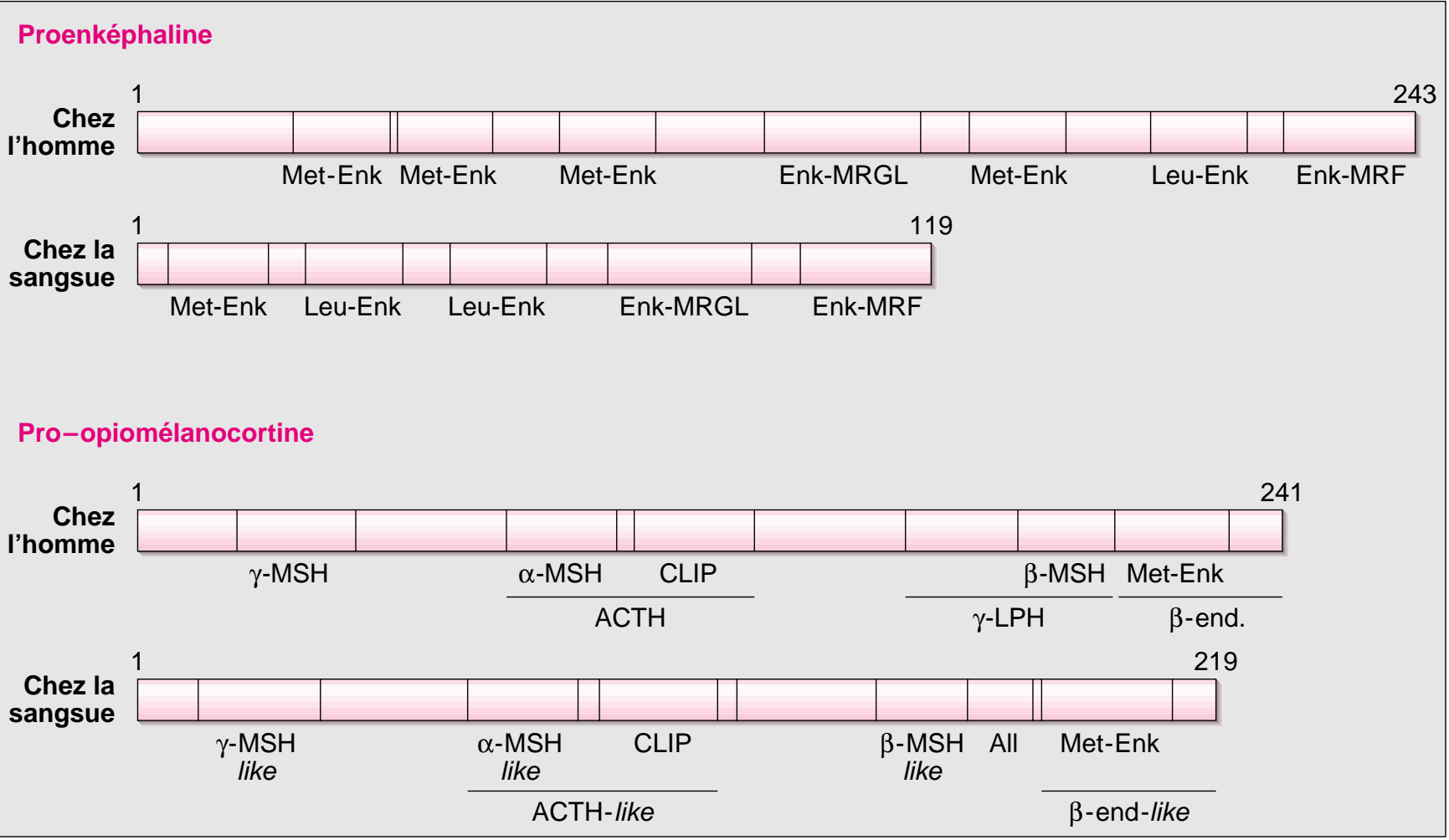

Figure 2. Structure des précurseurs de peptides opioïdes chez la sangsue Theromyzon tessulatum et chez I'homme $[3,4]$. La pro-enképhaline engendre par un processus de maturation des peptides opioïdes tels que la leucine enképhaline (Leu-Enk), la méthionine enképhaline (Met Enk) et d'autres molécules actives comme la méthionine enképhaline arginine-glycine-leucine (Enk-MRGL) et la méthionine enképhaline arginine-phénylalanine (Enk-MRF). La maturation de la pro-opiomélanocortine (POMC) entraîne également la synthèse de peptides opioïdes tels que la Met-Enk et la $\beta$-endorphine ( $\beta$-end). La comparaison entre les deux espèces révèle des différences notables dans la structure de ces deux précurseurs. En revanche, les produits de maturation sont sensiblement identiques (noter que les échelles relatives n'ont pas été respectées). 
et de ses produits de dégradation, stimule la prolifération des lymphocytes $\mathrm{T}$ cytotoxiques et des lymphocytes $\mathrm{T}$ auxiliaires [13]. La $\beta$-endorphine et la Met-Enk possèdent la capacité de stimuler in vitro la migration des neutrophiles, des monocytes et des lymphocytes $\mathrm{T}$ [10]. In vivo, des injections chroniques de ces deux peptides opioïdes entraînent la migration de ces mêmes cellules vers le site d'injection $[10,14]$. Notons que la Met-Enk peut, à forte concentration, avoir un effet immunorégulateur [10]. L'action de ces deux peptides passe par des récepteurs des opioïdes de type $\delta$, mis en évidence notamment au niveau des neutrophiles, des lymphocytes et des monocytes (figure 3) [10].

Ces peptides entraînent également la libération de cytokines participant à la réaction inflammatoire, telle que l'IL-6. Des travaux récents ont par ailleurs montré une augmentation des taux d'ARN messagers codant pour la pré-pro-enképhaline au niveau des monocytes de sang périphérique humain lorsque ceux-ci sont mis en présence de cytokines comme l'IL-6. Les cytokines et les peptides opiö̈des semblent donc avoir des actions étroitement liées dans les processus de mise en route de la réponse inflammatoire.

L'action biologique de ces peptides opioïdes est modulée par des endopeptidases de type endopeptidase neutre 24.11 (NEP)/CD10/enképhalinase. Cette enzyme, localisée sur la membrane plasmique des cellules inflammatoires humaines, assure en effet le catabolisme de la Met-Enk. Il est intéressant de noter que cette enzyme intervient aussi au niveau du système nerveux, comme enképhalinase et comme enzyme du catabolisme des neuropeptides [15].

Chez les invertébrés, les dérivés de la pro-enképhaline (Met-Enk, Leu-Enk et Met-Enk-RF) ont été caractérisés dans le système nerveux central [4]. Les dérivés de la POMC, tels que les $\gamma$-MSH et $\alpha$-MSH, ont été localisés respectivement à partir du système nerveux central d'annélides et d'insectes [16, 17].

Les précurseurs codant pour ces peptides ont eux aussi été mis en évidence dans le système nerveux. En effet, des études menées par immunocytochimie et hybridation in situ ont confirmé leur origine nerveuse. Cependant, leur caractérisation biochimique et moléculaire a été réali-

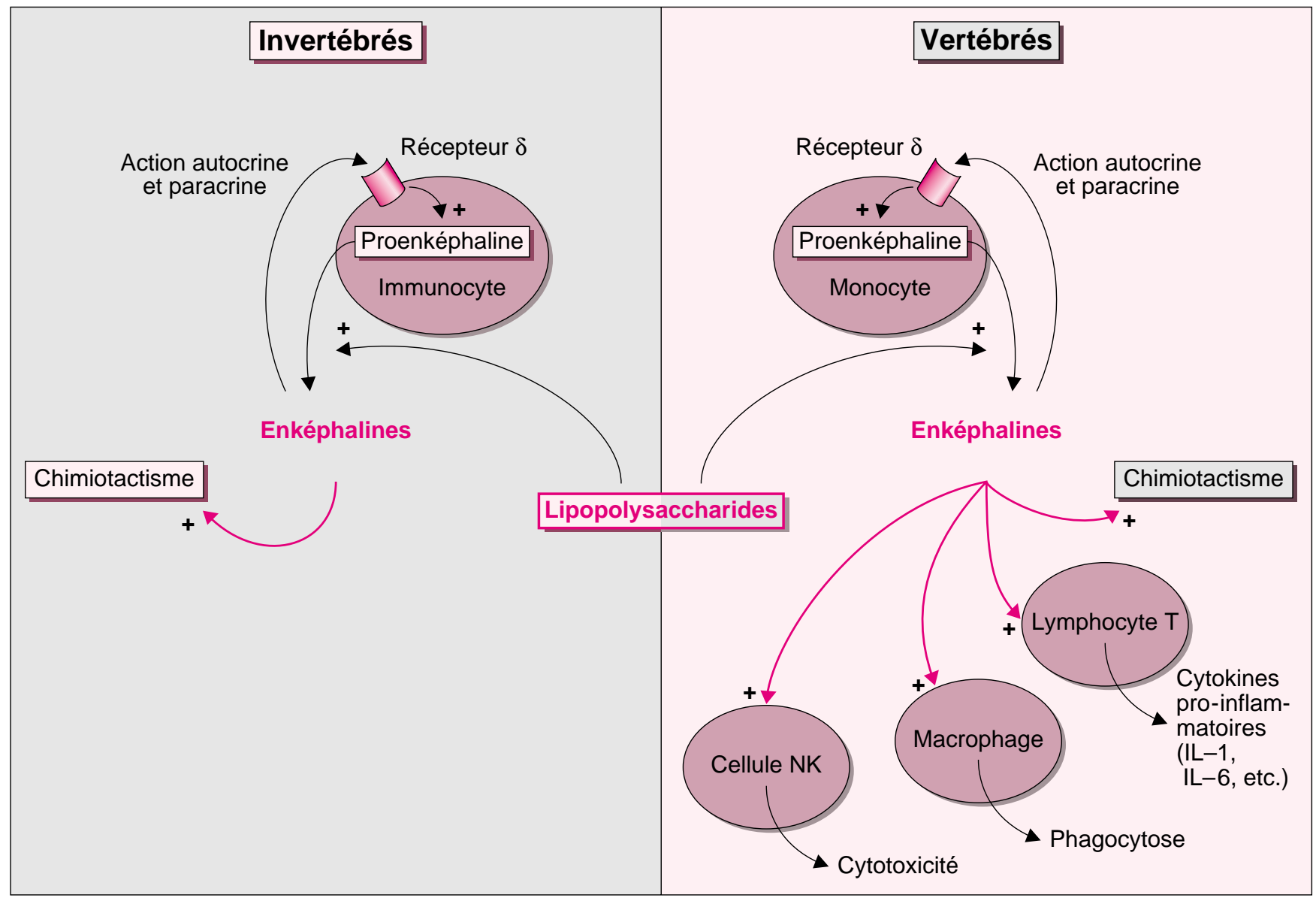

Figure 3. Comparaison des processus immunitaires, chez les vertébrés et les invertébrés, engendrés par la maturation de la pro-enképhaline, à la suite d'une injection de lipopolysaccharides (LPS). Une infection déclenchée expérimentalement entraîne la synthèse d'enképhalines. Ces peptides opioïdes, par leur action chimiotactique, vont mettre en route et entretenir la réaction inflammatoire. Ce précurseur présente donc un rôle important dans la réponse immunitaire innée. 
sée chez la sangsue à partir de cellules immunitaires (figure 2), et les mêmes résultats ont été obtenus chez la moule [18]. La particularité de ces précurseurs chez les invertébrés est la faible homologie de séquence avec celle des vertébrés. En revanche, les principes actifs tels que les enképhalines sont très conservés [19] et se situent dans la partie carboxy-terminale du précurseur, tout comme chez les vertébrés.

Ces analogies ont suscité l'étude de la maturation de la pro-enképhaline chez ces animaux. Des travaux réalisés chez la sangsue ont révélé qu'à la suite d'une stimulation par les lipopolysaccharides (LPS), la maturation démarre à l'extrémité carboxy-terminale en libérant la Met-Enk et la MetEnk-RF [4] dans le liquide cœlomique (fluide enveloppant les organes et dans lequel circulent les cellules immunitaires) (figure 3). Ces enképhalines sont capables d'activer les cellules immunitaires d'invertébrés [10, $14]$ en se fixant sur les récepteurs $\delta$ présents à leur surface. Cette activation se traduit à la fois par un changement de conformation et une migration vers le site inflammatoire en suivant un gradient de chémotaxie.

Les mécanismes enzymatiques impliqués dans la maturation des précurseurs chez les invertébrés sont encore mal connus. Les peptides opiö̈des sont réglés par des métallopeptidases telles que l'enzyme de conversion de l'angiotensine (ECA) et l'endopeptidase neutre 24.11 (NEP) qui peuvent, par clivage, engendrer des fragments plus petits. Ces derniers peuvent également être activateurs, mais ils peuvent aussi avoir perdu tout effet ou même être inhibiteurs du système immunitaire. A ce titre, l'ACTH(1-24) et l' $\alpha$-MSH (ou ACTH (1-13)) sont de bons exemples puisque le premier possède une action activatrice alors que le second est immunosuppresseur, bien que tous deux soient issus de l'ACTH (139) [2]. Les métallopeptidases sont présentes à la fois au niveau de la membrane plasmique des immunocytes et dans le liquide circulant [20]. Il existe actuellement un intérêt grandissant pour l'étude détaillée des mécanismes de l'immunité des invertébrés applicables aux cas des organismes supérieurs. Ainsi, la panoplie de peptides antimicrobiens présents chez les invertébrés constitue un élément majeur de l'immunité innée [21, 22]. Des peptides semblables sont également présents chez les vertébrés, telle la défensine [23]. Ces peptides paraissent donc bien adaptés pour offrir une première ligne de défense efficace contre les agents infectieux. Le deuxième exemple est illustré par les travaux récents de Medzhitov et al. [24] mettant en évidence la présence du récepteur Toll chez l'homme, initialement découvert chez la drosophile [25]. La découverte de ce récepteur, impliqué dans le déclenchement de la réponse immunitaire innée, démontre l'utilité de modèles d'étude plus simples. De tels résultats suggèrent que l'immunité innée est conservée des invertébrés jusqu'aux mammifères. Chez les vertébrés, ce type d'immunité pourrait permettre de lutter contre d'éventuelles infections pendant la mise en place de l'immunité acquise, puis de contrôler cette dernière par la production de signaux informant les cellules immunitaires de la présence d'agents pathogènes dans l'organisme. De nombreux travaux réalisés chez la drosophile font état de ces mécanismes de contrôle [25]. Des études menées en parallèle chez la sangsue et la moule ont permis d'appréhender les processus intervenant dans les minutes qui suivent l'infection, et donc impliqués dans la mise en route de la réponse immunitaire innée [19].

Par conséquent, chez les invertébrés comme chez les vertébrés, les peptides opioïdes sont des facteurs initiateurs de la réponse immunitaire innée (figure 4). De plus, ils entretiennent cette réaction et participent à la réaction immunitaire spécifique.

Le système immunitaire représente donc à tout moment le résultat d'un équilibre entre l'activation de la réponse immune (à la suite de la reconnaissance de molécules du non soi et/ou de l'entrée de pathogènes) et son rétrocontrôle, c'est-à-dire son inhibition (par des molécules telles que l' $\alpha$-MSH et les glucocorticoïdes). La rupture de cet équilibre peut conduire à de nombreuses maladies. Elles sont dues soit à une absence relative des mécanismes de rétrocontrôle (maladies auto-immunes), soit, à l'inverse, à leur exacerbation (SIDA [26], parasitose [27]).

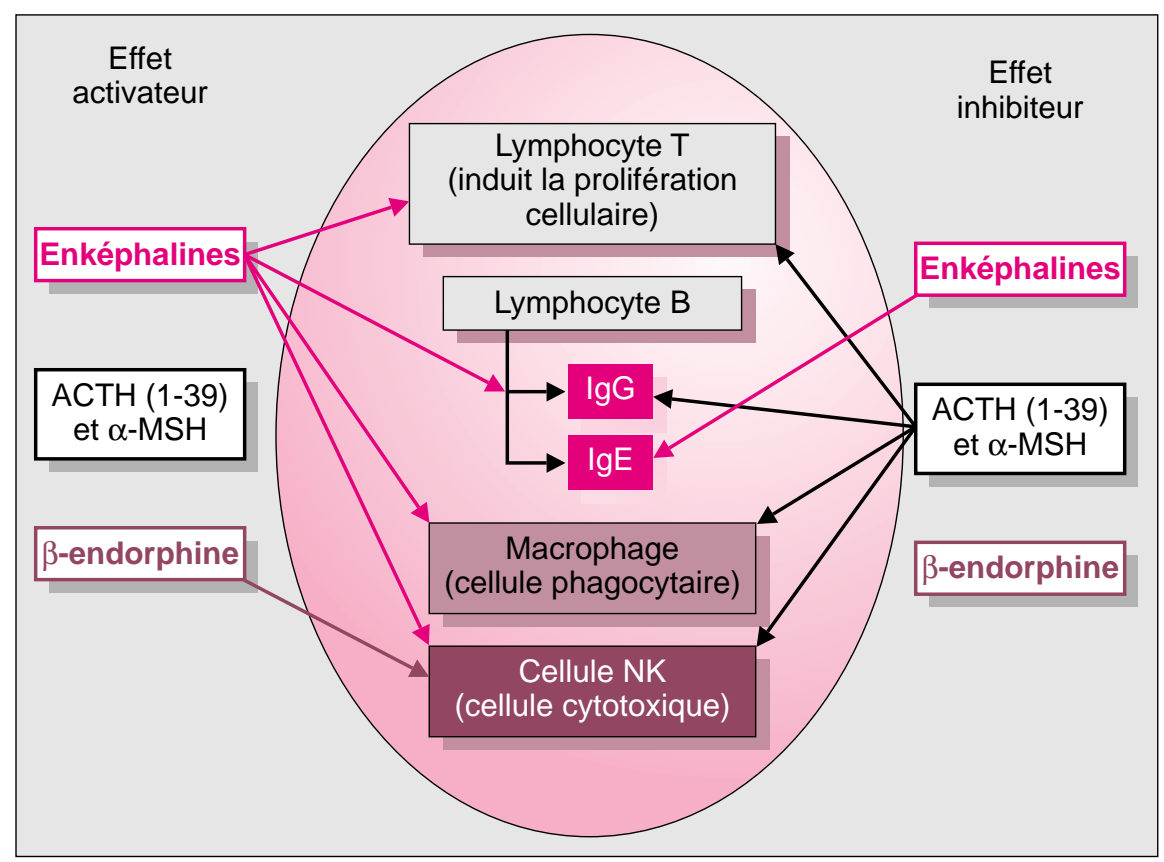

Figure 4. Modulation des réponses immunitaires innée et spécifique par les peptides opioïdes chez les vertébrés: actions activatrices et inhibitrices. L'effet activateur est assuré par les enképhalines issues de la pro-enképhaline et par la $\beta$-endorphine issue de la POMC. Ces peptides stimulent la réaction cytotoxique et la réponse humorale. À l'inverse, les dérivés de la POMC de type ACTH et $\alpha-M S H$ ont un effet inhibiteur. 


\section{Implication des substances opiacées dans la réponse immunitaire}

Les principales études ont porté sur les propriétés pharmacologiques de la morphine, qui est un opiacé alcaloïde exogène. Celle-ci fait partie, comme ses dérivés, de la famille des produits analgésiques. De nombreux travaux ont montré l'existence d'opiacés endogènes chez les invertébrés et les vertébrés [28], en particulier au niveau du tissu nerveux. L'intérêt s'est ainsi déplacé vers l'étude plus approfondie de ces messagers endogènes et de leurs rôles dans les conditions physiologiques normales ou pathologiques [29].

La synthèse d'un cannabinoïde (CB) endogène, l'anandamide, a été mise en évidence dans les systèmes nerveux et immunitaire d'invertébrés et de mammifères $[10,30]$. Des données récentes montrent que l'anandamide se fixe sur les cellules endothéliales des vaisseaux sanguins chez les mammifères [31]. Son action inhibitrice sur la réponse immune (figures 5 et 6) se produit via une famille de récepteurs $(\mathrm{CBr})$, composée de deux sous-types ( $\mathrm{CBr} 1$ et $\mathrm{CBr} 2)$.

Chez les invertébrés, le récepteur CBrl-like de l'anandamide a été mis en évidence dans les systèmes immunitaire et nerveux [32], confirmant ainsi l'origine très ancienne des cannabinoïdes. En outre, pour cette famille d'opiacés, des travaux réalisés sur des modèles invertébrés ont permis de comprendre certains mécanismes intervenant après la liaison entre le récepteur et son ligand. En effet, la transmision du signal intracellulaire implique la production de monoxyde d'azote (NO) par son enzyme de synthèse (cNOs) et l'activation de cette enzyme pourrait se faire à la suite d'une libération rapide du calcium présent dans le réticulum endoplasmique vers le cytoplasme (figure 6).

En ce qui concerne la morphine, son existence endogène suscite encore des controverses. L'hypothèse selon laquelle la dopamine serait un précurseur de la morphine dans certains neurones catécholaminergiques a été proposée depuis plus de dix ans par plusieurs équipes [10]. La synthèse

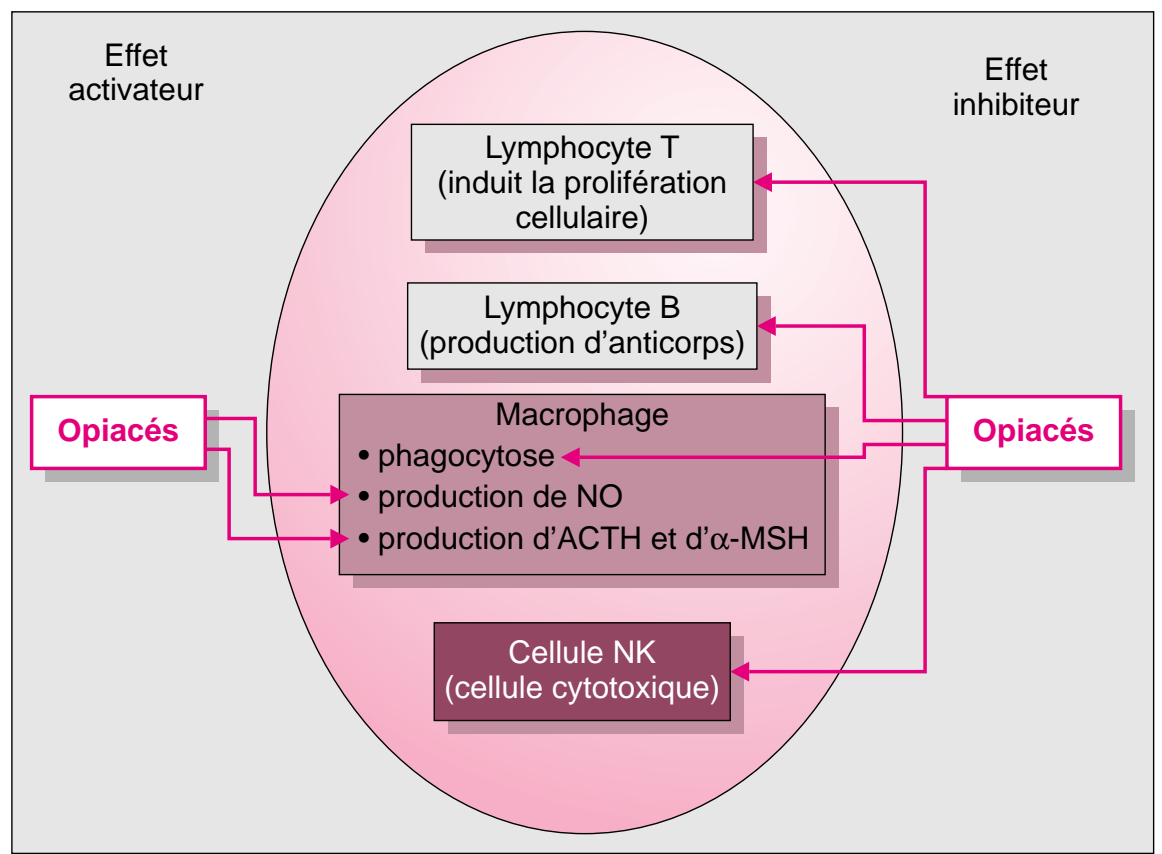

Figure 5. Modulation des réponses immunitaires innée et spécifique par les opiacés (morphine, anandamide) chez les vertébrés: actions activatrices et inhibitrices. Les opiacés inhibent la prolifération des lymphocytes, la réaction cytotoxique et la réponse humorale. En revanche, ils stimulent la production de certains dérivés de la POMC (ACTH et $\alpha-M S H)$ ainsi que la production de de la morphine n'a pas été démontrée dans les cellules immunitaires. En revanche, son action dans la modulation de la réponse inflammatoire a été récemment décrite.

En effet, chez les vertébrés, un traitement par la morphine engendre une inhibition de la mobilité des immunocytes, de leur prolifération dans les voies lymphatiques et de leur pouvoir phagocytaire. Elle atténue également fortement le phénomène de diapédèse en agissant sur les molécules d'adhérence des leucocytes (figure 5). Elle engendre également la libération d' $\alpha$-MSH (inhibiteur de la réponse immune) à partir de la POMC [11] dans les macrophages.

Chez les invertébrés, l'action immunorégulatrice de la morphine a également été démontrée. Celle-ci passe par une augmentation des métalloenzymes à la surface des immunocytes, assurant alors le clivage de l'ACTH en $\alpha$-MSH (figure 6) [16].

Chez les invertébrés et les vertébrés, la morphine agirait au niveau des immunocytes par un nouveau sous-type de récepteur: $\mu 3$ [10]. Sa présence a été démontrée sur les immunocytes d'invertébrés, sur les granulocytes humains [32] et sur l'endothélium des vaisseaux sanguins humains. Ce récepteur dérive d'un épissage alternatif du récepteur $\mu 1$. Son mécanisme intracellulaire serait analogue à celui des récepteurs $\mathrm{CBr}$ puisqu'il entraînerait la libération du calcium du réticulum endoplasmique et provoquerait à son tour une production massive de NO $[26,33]$.

Ces résultats montrent l'existence d'un processus anti-inflammatoire mettant en jeu les substances opiacées, le $\mathrm{NO}$ en tant que médiateur chimique, la POMC et le peptide immunorégulateur dérivé ( $\alpha-M S H)$. Ce mécanisme de contrôle s'effectue par les macrophages de façon autocrine et paracrine mais également par le système nerveux central par voie endocrine.

\section{Conclusions}

Cette voie de modulation du système immunitaire par les opiacés peut représenter un moyen de contrôle efficace contre "l'emballement " de la réaction inflammatoire chez l'homme. L'étude de cette modulation chez les invertébrés pré- 


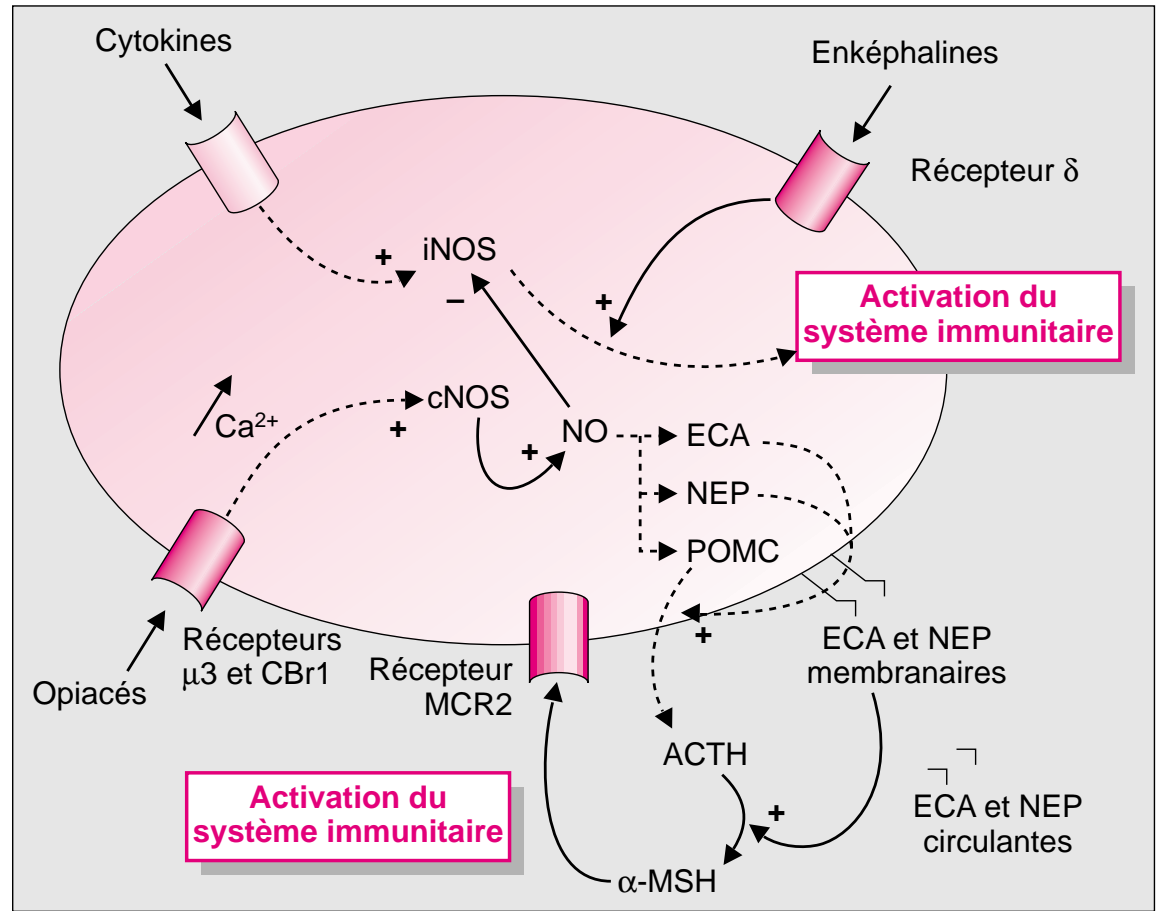

Figure 6. Mécanismes hypothétiques impliqués dans la modulation de la réponse immunitaire innée chez les vertébrés et les invertébrés. Les enképhalines et/ou les cytokines stimulent le système immunitaire en faisant intervenir la NO synthétase de type inductible (iNOs). À l'inverse, les opiacés, en se fixant sur leurs récepteurs respectifs, provoqueraient une augmentation de $\mathrm{Ca}^{2+}$ interne, qui conduit à la production de NO par la NO synthétase de type constitutive (cNOs). Le NO inhibe la réaction inflammatoire et active la libération de dérivés de la POMC (ACTH et $\alpha-M S H)$. Ces peptides inhibent de façon autocrine et paracrine la réponse immunitaire. Dans cette boucle d'inhibition, l'implication de métallopeptidases de type NEP et ECA, à la fois comme convertases et peptidases, est encore hypothétique. NEP: neutral endopeptidase, ECA: enzyme de conversion de l'angiotensine.

sente deux intérêts indéniables. Le premier est lié au fait que certains parasites (endo- ou exo-parasites) utilisent cette famille de molécules comme leurres envers le système immunitaire de l'hôte [27]. L'étude de la relation hôte-parasite via le détournement du système immunitaire de l'hôte par ces opiacés s'avère une voie d'étude fructueuse. Le second intérêt est d'ordre phylogénétique. En effet, la mise en évidence d'une modulation du système immunitaire par des messagers hormonaux apporte un éclairage nouveau sur la communication bidirectionnelle pouvant exister entre les systèmes immunitaire et nerveux.

Par conséquent, chez des animaux «primitifs» pouvant vivre plusieurs années, le système immunitaire paraît plus complexe et plus évolué qu'il n'avait été initialement pensé. De plus, des invertébrés jusqu'à

\section{RÉFÉRENCES}

1. Blalock JE, Smith EM. A complete regulatory loop between the immune and neuroendocrine systems. Fed Proc 1985; 44: 108-11.

2. Ottaviani E, Franceschi C. The neuroimmunology of stress from invertebrates to man. Prog Neurobiol 1996; 48: 421-40.

3. Patey G, Rossier J. Découverte, anatomie et biosynthèse des différentes familles de peptides opioïdes endogènes. Ann Endocrinol 1986; 47 : 71-87.

4. Stefano GB, Salzet M. Invertebrate opioid precursors: evolutionary conservation and the significance of enzymatic processing. Int Rev Cytol 1999; 187: 261-85.

5. Mechanick JI, Levin N, Roberts JL, Autelitano DJ. Proopiomelanocortin gene expression in a distinct population of rat spleen and lung leukocytes. Endocrinology 1992; 131: 518-25.

6. Kamphuis S, Eriksson F, Kavelaars A, et al. Role of endogenous pro-enkephalin A-derived peptides in human $\mathrm{T}$ cell proliferation and monocyte IL- 6 production. I Neuroimmunol 1998; 84: 53-60.

7. Marcinkiewicz M, Seidah NG, Chrétien M. Les convertases des prohormones et le système nerveux. Med Sci 1993; 9: 553-61.

8. LaMendola J, Martin SK, Steiner DF. Expression of PC3, carboxypeptidase E and enkephalin in human monocyte-derived macrophages as a tool for genetic studies. FEBS Lett 1997; 404: 19-22.

9. Weigent DA, Blalock JE. Production of peptide hormones and neurotransmitters by the immune system. Neuroimmunoendocrinology 1997 ; 69 : 1-30.

10. Stefano GB, Scharrer B, Smith EM, et al. Opioid and opiate immunoregulatory processes. Crit Rev Int Immunol 1996; 16: 109-44.

11. Lipton JM, Catania A. Anti-inflamatory actions of the neuroimmunomodulator $\alpha$ MSH. Immunol Today 1997; 18: 140-5.

12. Kowalski J. Immunologic action of [Met5] enkephalin fragments. Eur J Pharmacol 1998; 347: 95-9.

13. Sizemore RC, Dienglewicz RL, Pecunia E, Gottlied AA. Modulation of concannavalin A induced antigen-non specific regulatory cell activity by leu-enkephalin and related peptides. Clin Immunol Immunop 1991; $60: 310-8$.

14. Stefano GB, Shipp MA, Scharrer B. A possible immunoregulatory function for Met.-Enkephalin-Arg6-Phe7 involving human and invertebrate granulocytes. I Neuroimmunol 1991 ; 31 : 97-103.

15. Shipp MA, Stefano GB, D'Adamio L, et al. Downregulation of enkephalin-mediated inflammatory responses by $\mathrm{CD} 10 /$ neutral endopeptidase. Nature 1990; 247 : 394-6.

16. Salzet M, Salzet B, Cocquerelle C, et al. Biochemical and molecular chracterization of ACTH, its precursor and receptor in the leech Theromyzon tessulatum: morphine increases ACTH levels. J Immunol 1997; $159: 5400-11$. 


\section{RÉFÉRENCES}

17. Schoofs L, Jegou S, Vaudry H, Verhaert P, De Loof A. Localization of melanotropinlike peptides in the central nervous system of two insect species, the migratory locust, Locusta migratoria, and the fleshfly, Sarcophaga bullata. Cell Tissue Res 1987; 248: 25-31.

18. Ottaviani E, Franceschi C. The invertebrate phagocytic immunocyte: clues to a common evolution of immune and neuroendocrine systems. Immunol Today 1997; 18: 169-73.

19. Stefano GB, Salzet B, Fricchionne G. Enkelytin and opioid peptide association in invertebrates and vertebrates : immune activation and pain. Immunol Today 1998; 19: 243-89.

20. Laurent V, Stefano GB, Salzet M. Leech angiotensin converting enzyme. Trends Comp Endocrinol Neurobiol 1998; 839: 501-3.

21. Lemaitre B. La drosophile: un modèle pour l'étude de la réponse immunitaire innée. Med Sci 1999; 15: 15-22.

22. Bulet P. Les peptides antimicrobiens de la drosophile. Med Sci 1999; 15: 23-9.

23. Nicolas P, Mor A, Delfour A. Les peptides de la défense antimicrobienne des vertébrés. Med Sci 1992; 5 : 423-31.

24. Medzhitov R, Preston-Hulburt P, Janeway CA. A human homologue of the Drosophila Toll protein signals activation of adaptative immunity. Nature 1997; 388: 394-7.

25. Lemaitre B, Nicolas E, Michaut L, Reichart JM, Hoffmann JA. The dorsal regulatory gene cassette spätzle/Toll/cactus controls potent antifungal response in Drosophila adults. Cell 1997; 86: 973-83.

26. Stefano GB, Salzet M, Rialas CM, Mattocks D, Fimiani C, Bifinger TV. Macrophage behavior associated with acute and chronic to HIV GP120, morphine and anandamide: endothelial implications. Int J Cardiol 1998; 64: S3-13.

27. Capron A. Le langage moléculaire des parasites. Med Sci 1995; 11 : 431-41.

28. Lord JAH, Waterfield AA, Hughes J, Kosterlitz HW. Endogenous opioid peptides : multiple agonists and receptors. Nature 1977 ; 267 : 495-9.

29. Stefano GB, Digenis A, Spector S, et al. Opiate-like substances in an invertebrate, a novel opiate receptor on invertebrate and human immunocytes, and a role in immunosuppression. Proc Natl Acad Sci USA 1993; $90: 11099-103$

30. Klein TW, Newton C, Friedman H. Cannabinoid receptors and immunity. Immunol Today 1998; $19: 373-81$.

31. Klein J. Homology between immune responses invertebrates and invertebrates: does it exist? Scand J Immunol 1997; 46: 558-64.

32. Stefano GB, Liu Y, Goligorsky MS. Cannabinoid receptors are coupled to nitric oxide release in invertebrate immunocytes, microglia, and human monocytes. I Biol Chem 1996; 271 : 19238-42.

33. Stefano GB, Salzet M, Magazine H, Bilfinger TV. Anandamide or morphine stimulate saphenous vein endothelium cNOs nitric oxyde release which downregulates LPS and IFN- $\gamma$ iNOs by inhibiting adenylate cyclase. J Cardiol Pharmacol 1998; 31 : 813-20.

\section{TIRÉS À PART}

P. Chardin.

\section{Summary}

Opioids, opiates

and immune response

Opioids and opiates are involved both in the start and the inhibition of the immune response. Similarities concerning opioids precursors, their processing and the subsequent peptides have been shown in invertebrates and vertebrates. The subsequent peptides are involved in modulation of the immune system through their antibacterial activity and/or their capacity to induce inflammatory response. The inhibition of the immune response by opiates is highly conserved in course of evolution. The analogies between vertebrates and invertebrates underline an efficient and sophisticated immune strategy in which opioids and opiates play an important role. They appeared in invertebrate mechanisms that evolved at least 600 millions years ago. However, the complexity of nervous and immune systems makes difficult the study in mammals. Wherefore, another approach consists to develop invertebrates models. They are appropriate to delineate innate response mechanisms. These processes are all more important since vertebrate adaptive response generates from them.
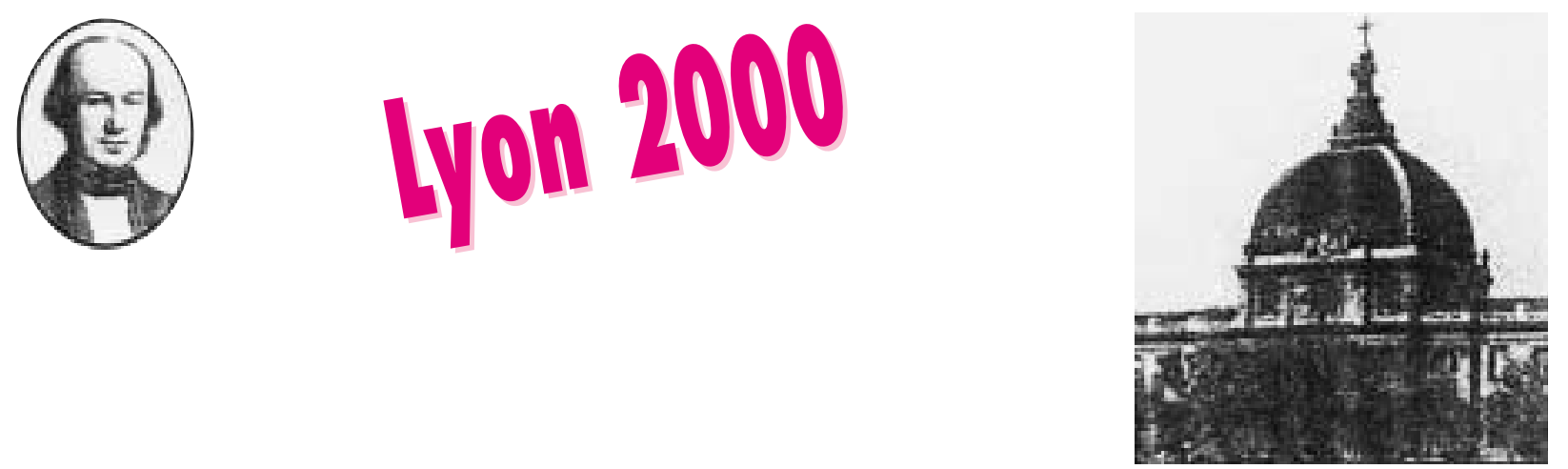